\title{
Highly Nuclear-Spin-Polarized Deuterium Atoms from the UV Photodissociation of Deuterium Iodide
}

\author{
Dimitris Sofikitis, ${ }^{1,2}$ Pavle Glodic, ${ }^{1,3}$ Greta Koumarianou, ${ }^{1,3}$ Hongyan Jiang, ${ }^{4}$ Lykourgos Bougas, ${ }^{1,2, *}$ \\ Peter C. Samartzis, ${ }^{1}$ Alexander Andreev, ${ }^{5,6}$ and T. Peter Rakitzis ${ }^{1,2, \uparrow}$ \\ ${ }^{1}$ Institute of Electronic Structure and Laser, Foundation for Research and Technology-Hellas, \\ 71110 Heraklion-Crete, Greece \\ ${ }^{2}$ Department of Physics, University of Crete, 70013 Heraklion-Crete, Greece \\ ${ }^{3}$ Department of Chemistry, University of Crete, 70013 Heraklion-Crete, Greece \\ ${ }^{4}$ Max Planck Institute for Biophysical Chemistry, Fassberg 11, D-37077 Göttingen, Germany \\ ${ }^{5}$ ELI-ALPS, 6720 Szeged, Hungary \\ ${ }^{6}$ Max Born Institute, 12489 Berlin, Germany \\ (Received 28 October 2016; published 8 June 2017)
}

\begin{abstract}
We report a novel highly spin-polarized deuterium (SPD) source, via the photodissociation of deuterium iodide at $270 \mathrm{~nm}$. I $\left({ }^{2} P_{3 / 2}\right)$ photofragments are ionized with $m$-state selectivity, and their velocity distribution measured via velocity-map slice imaging, from which the $\mathrm{D}$ polarization is determined. The process produces $\sim 100 \%$ electronically polarized $\mathrm{D}$ at the time of dissociation, which is then converted to $\sim 60 \%$ nuclear D polarization after $\sim 1.6 \mathrm{~ns}$. These production times for SPD allow collision-limited densities of $\sim 10^{18} \mathrm{~cm}^{-3}$ and at production rates of $\sim 10^{21} \mathrm{~s}^{-1}$ which are $10^{6}$ and $10^{4}$ times higher than conventional (Stern-Gerlach separation) methods, respectively. We discuss the production of SPD beams, and combining high-density SPD with laser fusion, to investigate polarized D-T, D- ${ }^{3} \mathrm{He}$, and D-D fusion.
\end{abstract}

DOI: 10.1103/PhysRevLett.118.233401

Nuclear-spin-polarized D atoms are important for the study of numerous nuclear reactions [1]. For example, controlling the nuclear spin polarization in fusion reactions offers important advantages, such as larger reaction cross sections, control over the emission direction of products, and in some cases suppressing unwanted neutron emission $[2,3]$. For the reactions $\mathrm{D}+\mathrm{T} \rightarrow n+{ }^{4} \mathrm{He}$ and $\mathrm{D}+{ }^{3} \mathrm{He} \rightarrow$ $p+{ }^{4} \mathrm{He}$, it is well known that the cross sections increase by $\sim 50 \%$ when the nuclei have oriented nuclear spins $[3,4]$. However, the experimental inability to produce spinpolarized deuterium (SPD) in a sufficient quantity has not allowed the realization of polarized fusion in a plasma. This lack of SPD has left the three most important questions of polarized fusion unanswered [3]:

(i) Does the nuclear polarization survive the plasma long enough to benefit fusion? Theoretical calculations indicate that it likely does [5,6]; however, an experimental demonstration is lacking (although proposals for such a demonstration have been made recently $[7,8]$ ).

(ii) What is the polarization dependence of the D-D fusion reaction? Over the past 30 years, several theoretical predictions, from 10 to $100 \mathrm{keV}$, range from enhancement, suppression, or no effect on reactivity [9-14], leaving a long-standing need for experimental resolution.

(iii) Assuming (i) is positive, can sufficient SPD be produced for a nuclear reactor, $\sim 10^{21} \mathrm{~s}^{-1}$ [3]? For current methods, either the SPD production rate or polarization is too low. For example, large-scale magnetic (SternGerlach) separators are limited to production rates of $\sim 10^{17}$ atoms $\mathrm{s}^{-1}$ [3], and polarized solid deuterium targets are produced where densities reach $\sim 3 \times 10^{19}$ spins $/ \mathrm{cm}^{3}$ but at a low polarization of $\sim 10 \%$ [15].

In this Letter, we demonstrate the production of SPD from the photodissociation of deuterium iodide, which can offer sufficient SPD densities and production rates to help answer open questions (i)-(iii). Magnetic separation methods require low beam densities to avoid collisions on the millisecond time scale of the separation process, which limits densities to $\sim 10^{12} \mathrm{~cm}^{-3}$ [3]. In contrast, the DI photodissociation method requires only $1.6 \mathrm{~ns}$ (the D-atom hyperfine-beating time) to polarize the $\mathrm{D}$ nuclear spin, which is $10^{6}$ times faster than magnetic separation, allowing densities larger by a similar factor, of the order of $10^{18} \mathrm{~cm}^{-3}$. In addition, one photodissociating photon produces one SPD nucleus; industrial-scale kilowatt UV lasers can therefore achieve SPD production rates of at least $10^{21} \mathrm{~s}^{-1}$. Below, we discuss how SPD pulsed densities of $\sim 10^{18} \mathrm{~cm}^{-3}$ can be combined with pulsed laser fusion to study D-T, D- ${ }^{3} \mathrm{He}$, and D-D polarized reactions.

Laser photodissociation of hydrogen halides has been shown to produce highly polarized $\mathrm{H}$ atoms [16-20]. The photodissociation process initially polarizes the electron spin, up to $100 \%$ (for photofragment velocities parallel to the propagation direction of the circularly polarized dissociation laser light) [21]; due to the hyperfine interaction, the polarization oscillates back and forth between the electronic and the nuclear spin on the $\sim 1$-ns time scale. By terminating this polarization exchange appropriately, for example, by ionizing the atom, one can isolate the 
polarization in the electronic or nuclear spin. This pulsed production of hyperpolarized $\mathrm{H}$, here extended to the production of hyperpolarized $\mathrm{D}$ atoms, can be used to study polarized D fusion reactions at a high density, with much larger signals than conventional beam methods. The $\mathrm{D}$ atoms can be used to produce pulsed ion beams, allowing the possibility of measuring polarized fusion reaction cross sections over a large energy range. The pulsed production can also be combined with inertial confinement fusion, where fusion is induced by an intense short laser pulse [22]. The photolysis and fusion pulses can be timed so that the D nuclei are maximally polarized when fusion occurs; such a combination can offer a straightforward method for measuring the effect of polarization in the $\mathrm{D}$ reactions, at densities close to $10^{18} \mathrm{~cm}^{-3}$ and at collision energies of $\sim 10 \mathrm{keV}$.

Theoretical calculations of the photofragment polarizations, over a range of UV photodissociation energies, have been performed for nearly all the hydrogen halides: HF/DF [23], $\mathrm{HCl} / \mathrm{DCl}$ [24], $\mathrm{HBr}$ [25], and HI/DI [26]. At least two competing dissociation mechanisms occur: excitation to a dissociative state followed by adiabatic dissociation producing hydrogen atoms with spin-down electrons and excitation to another dissociative state followed by adiabatic dissociation producing hydrogen atoms with spin-up electrons (also nonadiabatic transitions between dissociative states can change the $\mathrm{H}$-atom polarization). Therefore, depending on the contribution from each mechanism, the hydrogen electronic spin polarization $P_{e}$ can range from fully spin up $\left(P_{e}=1\right)$ to fully spin down $\left(P_{e}=-1\right)$, and anything in between, including unpolarized $\left(P_{e}=0\right)$. Calculations predict that $P_{e}$ can take on values over this complete range and depends strongly on the photolysis energy, on the halide cofragment $(\mathrm{F}, \mathrm{Cl}, \mathrm{Br}$, or I), and on the hydrogen isotope. For example, near maximal $\mathrm{H}$ polarizations $\left(\left|P_{e}\right|=1\right)$ are calculated at some energies for photodissociation of the hydrogen halides $\mathrm{HCl}, \mathrm{HBr}$, and $\mathrm{HI}$ (of which $\mathrm{HCl}$ and $\mathrm{HBr}$ have been measured at $193 \mathrm{~nm}$ $[16,18]$ ) and the deuterium halides DF and DI (which have not been measured previously). Here we determine the polarization of the $\mathrm{D}$ and I photofragments from the photodissociation of DI molecules at $270 \mathrm{~nm}$, demonstrating a source of highly polarized high-density D atoms.

The experiments are performed using a molecular beam setup, to facilitate polarization-sensitive detection. We measure the spin polarization of the iodine atoms via a resonance-enhanced multiphoton ionization (REMPI) scheme and infer the deuterium polarization by angular momentum conservation; past experiments of direct measurements of hydrogen spin polarization give excellent agreement with indirect detection [19,20,27,28].

The experimental setup has been explained in detail elsewhere $[29,30]$. Briefly, deuterium iodide is mixed with $\mathrm{He}$ in a $50 \%$ ratio and expanded into the chamber via a pulsed nozzle. The molecular beam is intersected at right angles $\sim 50 \mathrm{~mm}$ downstream by the focused photolysis
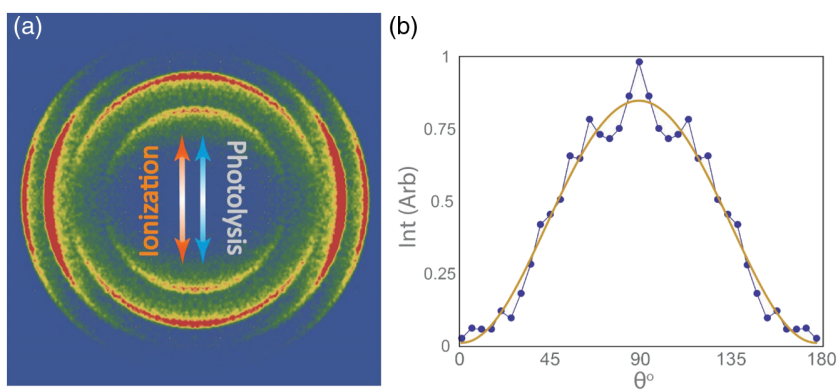

FIG. 1. (a) Sliced ion image of the D produced by DI photodissociation. The photolysis and ionization lasers counterpropagate along the $90^{\circ}-270^{\circ}$ direction, while the direction of their linear polarization is marked by the arrows. (b) Angular distribution of the ions corresponding to DI $+270 \mathrm{~nm} \rightarrow \mathrm{D}+\mathrm{I}\left({ }^{2} P_{3 / 2}\right)$ (points) and the fit with Eq. (3) (orange solid line).

and probe laser beams. The resulting ion sphere is focused by a single ion lens on a gated position-sensitive detector, thus performing velocity map imaging [31].

Figure 1 shows an image of the $\mathrm{D}$ ions produced by dissociation of DI at 270 and $243 \mathrm{~nm}$ in coincidence with $\mathrm{I}\left({ }^{2} P_{3 / 2}\right)$ and $\mathrm{I}\left({ }^{2} P_{1 / 2}\right)$ (henceforth denoted as I and $\mathrm{I}^{*}$, respectively). The $\mathrm{D}$ photofragments are ionized via $2+1$ REMPI at $243 \mathrm{~nm}$. From inner to outer, the four rings correspond to dissociation channels: (1) DI $+270 \mathrm{~nm} \rightarrow$ $\mathrm{D}+\mathrm{I}^{*}\left(v_{D}=7242 \mathrm{~m} / \mathrm{s}\right),(2) \mathrm{DI}+243 \mathrm{~nm} \rightarrow \mathrm{D}+\mathrm{I}^{*}\left(v_{D}=\right.$ $10184 \mathrm{~m} / \mathrm{s})$, (3) DI $+270 \mathrm{~nm} \rightarrow \mathrm{D}+\mathrm{I}\left(v_{D}=12032 \mathrm{~m} / \mathrm{s}\right)$, and (4) DI $+243 \mathrm{~nm} \rightarrow \mathrm{D}+\mathrm{I}\left(v_{D}=13880 \mathrm{~m} / \mathrm{s}\right)$, where $v_{D}$ is the velocity of the $\mathrm{D}$ atoms. Without the probe field at $243 \mathrm{~nm}$, only channels (1) and (3) are present. Channel (1) is accessed by a parallel transition to the $a^{3} \Pi_{0^{+}}$state and does not yield SPD:

$\mathrm{DI}\left(\Omega_{a}=0\right) \rightarrow \mathrm{D}\left(m_{S}= \pm 1 / 2\right)+\mathrm{I}^{*}\left(m_{J}=\mp 1 / 2\right)$.

Channel (3) is accessed by a perpendicular transition to the $A^{1} \Pi_{1}$ state and yields SPD:

$\mathrm{DI}\left(\Omega_{A}= \pm 1\right) \rightarrow \mathrm{D}\left(m_{S}=\mp 1 / 2\right)+\mathrm{I}\left(m_{J}= \pm 3 / 2\right)$.

Circularly polarized photolysis light $\sigma^{+}$excites the $\Omega_{A}=+1$ state (or $\sigma^{-}$excites the $\Omega_{A}=-1$ state), only for photofragment recoil direction $\hat{v}$ parallel to the propagation direction $\hat{k}\left(\theta_{v k}=0\right.$; see Fig. 2). Otherwise, a coherent superposition of the two $\Omega_{A}= \pm 1$ states is excited, and SPD polarization falls as $\hat{v} \cdot \hat{k}=\cos \theta_{v k}$. This loss in polarization can be minimized by aligning the DI bonds using a strong nonresonant laser pulse [32,33], along $\hat{k}$ just before photodissociation, which also eliminates the unwanted unpolarized $D+\mathrm{I}^{*}$ channel (1).

The angular distribution of the polarized photofragments along the quantization axis can be expressed as an even 


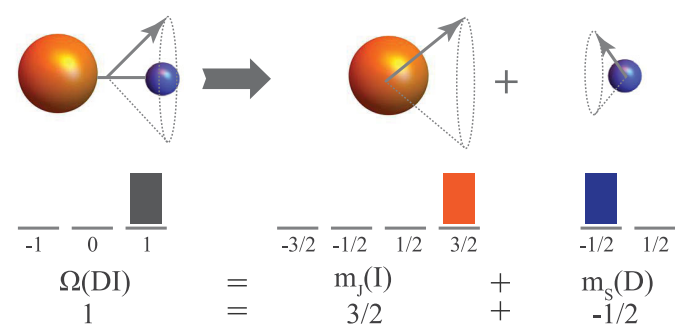

FIG. 2. The angular momentum projection $\Omega_{A}=+1$ is prepared from the DI ( $\Omega=0$ ) ground state, using $\sigma^{+}$circularly polarized photolysis light, and is distributed to the angular momentum projections of the photofragments after photodissociation.

expansion of Legendre polynomials [32,34] up to the fourth order:

$$
\mathrm{I}(\theta) / \mathrm{I}_{0}=1+\beta_{2} P_{2}(\cos \theta)+\beta_{4} P_{4}(\cos \theta),
$$

where $P_{n}(x)$ is the $n$ th-order Legendre polynomial. Figure 1(b) depicts the angular distribution of the deuterium ions correlated with the production of $\mathrm{I}$ atoms at $270 \mathrm{~nm}$, as well as a fit using Eq. (3), for which $\beta_{2}=\beta$ and $\beta_{4}=0$. The value of the spatial-anisotropy $\beta$ parameter extracted by this fit is $\beta=-0.98 \pm 0.03$, corresponding to an almost purely perpendicular transition, in agreement with theoretical predictions [26].

Figures 3(a)-3(e) show sliced ion images of I atoms produced by $\mathrm{DI}+270 \mathrm{~nm} \rightarrow \mathrm{D}+\mathrm{I}$ and detected via the ${ }^{2} P_{3 / 2} \rightarrow{ }^{4} P_{1 / 2}$ REMPI transition at $303.6 \mathrm{~nm}$. The intensities of the $6 \mathrm{~ns}$ dissociation and ionization laser pulses were $\sim 1$ and $\sim 0.5 \mathrm{~mJ}$, respectively, and both were focused with $f=30 \mathrm{~cm}$ lenses. In Fig. 3(a), both the photolysis and the ionization laser pulses are right-circularly polarized (RCP); the angular distribution is strongly anisotropic, with most of the photofragments recoiling preferentially along the propagation direction of the laser beams. For Fig. 3(b), the photolysis laser has the opposite helicity: left-circularly polarized (LCP), and we see a very big change in the angular distribution, as it is nearly isotropic. The ratio of the total intensities of the images in Figs. 3(a) and 3(b) is $\mathrm{I}_{0}(R R) / \mathrm{I}_{0}(L R)=1.8 \pm 0.2$. Together, these measurements show that the I atoms are strongly polarized. Figures 3(c)-3(e) show images using linearly polarized photolysis and ionization lasers, aligned in the geometries $(X X),(X Z)$, and $(Z X)$, respectively.

The data from Fig. 3 are analyzed to determine the spherical-basis density matrix elements $a_{q}^{(k)}(p)$ [35], for the I atoms, using established methods $[16,18,36]$ and hyperfine depolarization coefficients $\left\langle G^{(1)}\right\rangle \approx 0.400$ and $\left\langle G^{(2)}\right\rangle \approx$ 0.233. In Fig. 4, we present graphically the fitted values of the $\beta$ parameter, for the $\mathrm{D}$ and I photofragments, as well as the $a_{q}^{(k)}(p)$ parameters for $\mathrm{I}\left({ }^{2} P_{3 / 2}\right)$, along with the corresponding values of $\left\langle m_{J}(\mathrm{I})\right\rangle$ and the inferred D-atom electron spin projection $\left\langle m_{S}(\mathrm{D})\right\rangle=1-\left\langle m_{J}(\mathrm{I})\right\rangle$. The gray
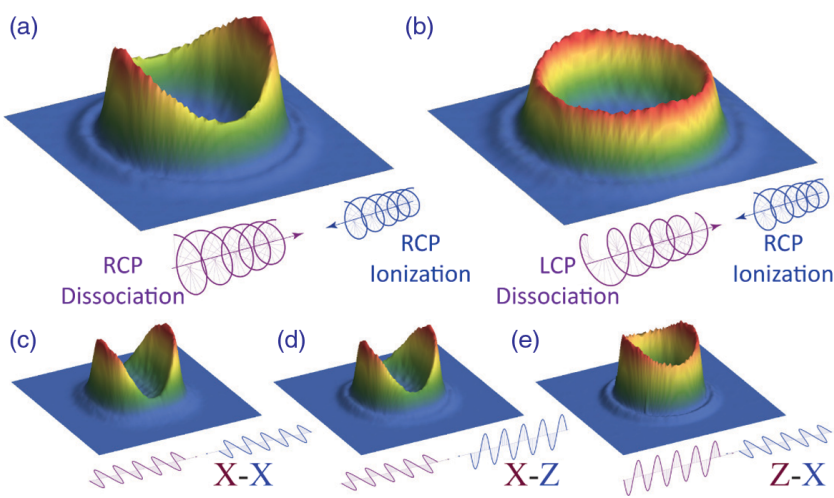

FIG. 3. Sliced ion image of I atoms produced with the polarizations of the photolysis laser $(\lambda=270 \mathrm{~nm})$ and the ionization laser $(\lambda=303.6 \mathrm{~nm}$ ) being (a) both right-circularly polarized (RCP), (b) left-circularly polarized (LCP) and RCP, (c) both linearly polarized parallel to the image plane $X$, (d) linearly polarized along $X$ and linearly polarized perpendicular to the image plane $Z$, and (e) linearly polarized along $Z$ and linearly polarized along $X$.

histograms represent the physical ranges of these parameters. The value of each of the $a_{0}^{(1)}(\perp)$ and $a_{0}^{(2)}(\perp)$ parameters is maximal, meaning that $\left\langle m_{j}(\mathrm{I})\right\rangle=1.5$ must also be maximal, constraining the $\mathrm{D}$ electron spin polarization to be maximally polarized in the opposite direction, $\left\langle m_{S}(\mathrm{D})\right\rangle=-0.5$ [26], as shown in Fig. 2. These $m$-state values are explained by Eq. (2).

The measured $\beta$ and $a_{q}^{k}(p)$ parameters are consistent with those of the dissociation mechanism predicted at a significantly higher energy, $\sim 47000 \mathrm{~cm}^{-1}$ (Fig. 4) [26]. However, at lower energies, the dissociation is predicted to switch to excitation and adiabatic dissociation via the $a^{3} \Pi_{1}$ state, producing $m_{J}(\mathrm{I})=+1 / 2$ and $m_{S}(\mathrm{D})=+1 / 2$ [26]; the energy of this mechanism switch, predicted between

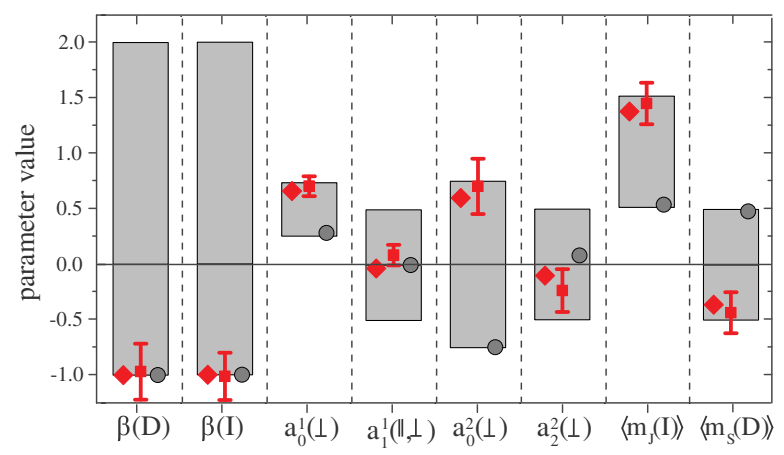

FIG. 4. Results for the $\beta$ parameter for the D and I atoms from the photodissociation of DI at $37000 \mathrm{~cm}^{-1}$ and the I $a_{q}^{(k)}(p)$ parameters, which give $\left\langle m_{J}(\mathrm{I})\right\rangle \approx 1.5$ and $\left\langle m_{S}(\mathrm{D})\right\rangle \approx-0.5$. Error bars are $2 \sigma$ confidence intervals, and the gray bars give the allowed range of the parameters. Theoretical predictions [23] are given for energies of 47000 (red diamonds) and $37000 \mathrm{~cm}^{-1}$ (gray circles). 


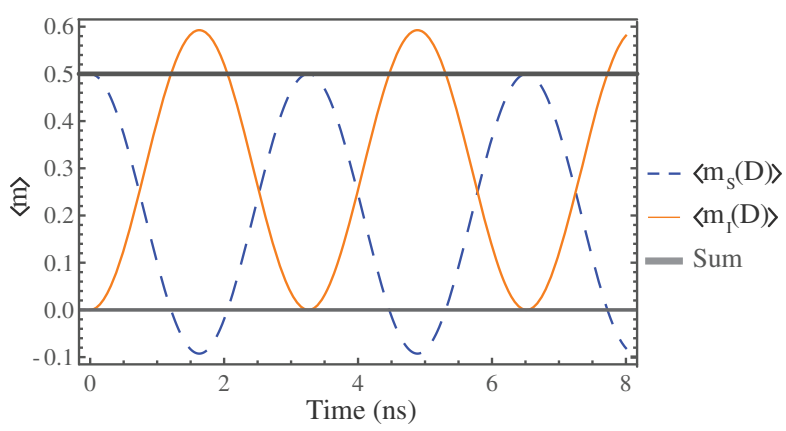

FIG. 5. Evolution of the spin projection expectation values of the electron $\left\langle m_{S}(\mathrm{D})\right\rangle$ (dashed line), the nucleus $\left\langle m_{I}(\mathrm{D})\right\rangle$ (thin solid line), and the sum (thick solid line) of the $\mathrm{D}$ atom due to the hyperfine interaction.

37000 and $44000 \mathrm{~cm}^{-1}$, is strongly model dependent [37]. Our results at $\sim 37000 \mathrm{~cm}^{-1}$ do not show this mechanism switch, indicating that the dissociation models may need to be modified.

The photodissociation process initially polarizes the electron spin $\mathbf{S}$ leaving the nuclear spin $\mathbf{I}$ initially unpolarized. The $\mathrm{D}$ atoms are found in a coherent superposition of the total angular momentum states $\left|F, M_{F}\right\rangle$, defined by the coupling $\mathbf{F}=\mathbf{S}+\mathbf{I}$, which are the eigenstates of the system. Therefore, the system evolves in time, transferring the polarization of the electron spin to the nuclear spin and back [38]. Such a polarization transfer has been experimentally demonstrated in several cases $[39,40]$. Here, the polarization transfer can be quantified [41] using Eqs. (3)-(6) in Ref. [42], giving

$$
\begin{aligned}
& \left\langle m_{S}(\mathrm{D})\right\rangle=\frac{16}{27} \sin ^{2}\left(\frac{\Delta E}{2 \hbar} t\right), \\
& \left\langle m_{I}(\mathrm{D})\right\rangle=\frac{1}{2}-\frac{16}{27} \sin ^{2}\left(\frac{\Delta E}{2 \hbar} t\right),
\end{aligned}
$$

where $\Delta E=327.37 \mathrm{MHz}$ is the hyperfine splitting in the deuterium atom [43]. We show the polarization dependence of the D electron and nuclear spin, by plotting Eqs. (4a) and (4b) in Fig. 5. At $t=0$, the electron spin is fully polarized, and the nuclear spin is unpolarized. The electron spin polarization is transferred to the nuclear spin and back, with a period of $3.2 \mathrm{~ns}$. An interesting aspect of these dynamics is that there exist times (for example, around 1.6 and $4.8 \mathrm{~ns}$ ) where the electron spin polarization is opposite to the initial one, albeit with a much smaller value. At the same times, the spin projection of the $\mathrm{D}$ nucleus reaches a value of $\sim 60 \%$.

The main depolarizer of the $\mathrm{D}$ atoms will be the halogenatom cofragment. Thermal spin-exchange rates of H/D with halogen atoms are not available but are $\sim 10^{-9} \mathrm{~cm}^{3} \mathrm{~s}^{-1}$ for collisions with alkali atoms [44]. Using this rate, maximal densities of $\sim 10^{18} \mathrm{~cm}^{-3}$ of spin-polarized $\mathrm{D}$ atoms can be produced over the needed time of $\sim 1 \mathrm{~ns}$. However, for the photodissociation of DF near $193 \mathrm{~nm}$, it is predicted that both the $\mathrm{D}$ and the $\mathrm{F}\left({ }^{2} P_{3 / 2}\right)$ cofragment will have the same polarization: $\left\langle m_{S}(\mathrm{D})\right\rangle=\left\langle m_{J}(\mathrm{~F})\right\rangle=0.5$; there is even the possibility of polarizing the $\mathrm{F}\left({ }^{2} P_{3 / 2}\right)$ further via infrared excitation of the DF before photodissociation [38-42]. As all colliding species will be similarly polarized, the depolarization rate of SPD should be significantly reduced, and perhaps densities greater than $\sim 10^{18} \mathrm{~cm}^{-3}$ can be produced; e.g., densities of spin-polarized $\mathrm{O}_{2} \sim 10^{19} \mathrm{~cm}^{-3}$ were reported recently [45].

Laser-fusion neutron-yield calculations were performed using a modified [46] MEDUSA code [47], which include the following physical processes: initial laser pulse absorption, propagation of a heat wave, ionization, one-dimensional hydrodynamics of plasma by two-temperature approximation, nonlinear thermal conductivity and limitation, classical and resonant absorption of laser radiation, thermal radiation emission and its absorption by plasma, and thermonuclear reaction yield. The calculations predict that the irradiation of $10^{18} \mathrm{~cm}^{-3}$ deuterium and tritium atoms and $10^{19} \mathrm{~cm}^{-3}{ }^{3} \mathrm{He}$ with a $2 \mathrm{MJ}, 2.3 \mathrm{~ns}$ pulse at $350 \mathrm{~nm}$ focused to $\sim 100 \mu \mathrm{m}$ (National Ignition Facility at Lawrence Livermore National Laboratory) will heat the resulting ions to average collision (thermal) energies of $\sim 10 \mathrm{keV}$ and lead to the production of $\sim 10^{7}$ neutrons/pulse from the D-T reaction and $\sim 10^{5}$ neutrons/pulse each from the D-D and D- ${ }^{3} \mathrm{He}$ reactions. Irradiation with a $6 \mathrm{~kJ}$ (with $\lambda \sim 1 \mu \mathrm{m}$ ), $1 \mathrm{ps}$ laser pulse [48,49], focused to $10 \mu \mathrm{m}$, will produce $\sim 10^{4}$ neutrons/pulse from D-T reactions and $\sim 100$ neutrons/pulse from $\mathrm{D}-\mathrm{D}$ or $\mathrm{D}-{ }^{3} \mathrm{He}$ fusion reactions. These neutron yields compare well with the ones foreseen for ongoing polarized fusion experiments, of $\sim 0.01$ neutrons/s [50], and show that the study of polarized fusion with high signals is possible, using high-density SPD and laser-initiated fusion.

Highly polarized D, and likely $\mathrm{T}$, can be produced by deuterium and tritium halide photodissociation, respectively, using picosecond photodissociation and ionization lasers (DI photodissociation is saturated by $5 \mathrm{~mJ} /$ pulse $270 \mathrm{~nm}$, focused to $100 \mu \mathrm{m}$ ). Highly polarized ${ }^{3} \mathrm{He}$ can be produced by spin-exchange optical pumping [51,52]. Subsequently, using the conditions described above, laser fusion of D-T or D- ${ }^{3} \mathrm{He}$ will give neutron or proton reaction products with angular distributions of the form $a+\sin ^{2} \theta$ about the spin quantization axis [5] (where $a=0$ for fully polarized spins; for $\mathrm{D}$ polarization $p_{z}=0.55$ and $p_{z z}=0$ [50], $a \sim 1$ for the reaction with T polarization of $p_{z}=0.95$, and $a \sim 2$ for ${ }^{3} \mathrm{He}$ with $p_{z}=0.76$ [53]). Such large spatial anisotropy contrasts can determine whether spin polarization survives laser plasma [question (i)]. In addition, the neutron products will be spin polarized [5] and can be measured with neutron polarimeters [54]. Finally, polarized D-D reaction cross sections can be measured by comparing (in a single laser shot) the ratios of the total signal and angular distributions of the D-D and D-T (or D- ${ }^{3} \mathrm{He}$ ) reaction products, for the cases of polarized, and unpolarized, reactants [question (ii)]. 
The experimental work was conducted at the Ultraviolet Laser Facility at FORTH-IESL, supported in part by the European Union's Horizon 2020 research and innovation programme LASERLAB-EUROPE (Grant Agreement No. 654148), and the European Research Council (ERC) Grant TRICEPS (GA No. 207542).

*Present address: Johannes Gutenberg-Universität Mainz, 55128 Mainz, Germany. †ptr@iesl.forth.gr

[1] E. Steffens and W. Haeberli, Rep. Prog. Phys. 66, 1887 (2003).

[2] G. Ciullo, Polarized Fusion: An Idea More Than Thirty Years Old! What Are We Waiting For? (Springer, New York, 2016), pp. 1-13.

[3] R. Engels et al., Phys. Part. Nucl. 45, 341 (2014).

[4] C. Leemann, H. Bürgisser, P. Huber, U. Rohrer, H. Paetz gen. Schieck, and F. Seiler, Ann. Phys. (N.Y.) 66, 810 (1971).

[5] R. M. Kulsrud, H. P. Furth, E. J. Valeo, and M. Goldhaber, Phys. Rev. Lett. 49, 1248 (1982).

[6] R. M. More, Phys. Rev. Lett. 51, 396 (1983).

[7] J.-P. Didelez and C. Deutsch, Laser Part. Beams 33, 211 (2015).

[8] D. C. Pace, M. J. Lanctot, G. L. Jackson, A. M. Sandorfi, S. P. Smith, and X. Wei, J. Fusion Energy 35, 54 (2016).

[9] K. A. Fletcher, Z. Ayer, T. C. Black, R. K. Das, H. J. Karwowski, E. J. Ludwig, and G. M. Hale, Phys. Rev. C 49, 2305 (1994).

[10] G. Hale and G. Doolen, Los Alamos Report No. LA-9971MS, 1984.

[11] H. M. Hofmann and D. Fick, Phys. Rev. Lett. 52, 2038 (1984).

[12] S. Lemaître and H. Paetz gen. Schieck, Ann. Phys. (Berlin) 505, 503 (1993).

[13] J. S. Zhang, K. F. Liu, and G. W. Shuy, Phys. Rev. Lett. 57, 1410 (1986).

[14] J. S. Zhang, K. F. Liu, and G. W. Shuy, Phys. Rev. C 60, 054614 (1999).

[15] M. Iio et al., Nucl. Instrum. Methods Phys. Res., Sect. A 526, 190 (2004).

[16] T. P. Rakitzis, P. C. Samartzis, R. L. Toomes, T. N. Kitsopoulos, A. Brown, G. G. Balint-Kurti, O. S. Vasyutinskii, and J. A. Beswick, Science 300, 1936 (2003).

[17] T. P. Rakitzis, ChemPhysChem 5, 1489 (2004).

[18] T. P. Rakitzis, P. C. Samartzis, R. L. Toomes, and T. N. Kitsopoulos, J. Chem. Phys. 121, 7222 (2004).

[19] D. Sofikitis, L. Rubio-Lago, L. Bougas, A. J. Alexander, and T. P. Rakitzis, J. Chem. Phys. 129, 144302 (2008).

[20] D. Sofikitis, L. Rubio-Lago, A. J. Alexander, and T. P. Rakitzis, Europhys. Lett. 81, 68002 (2008).

[21] R. J. Van Brunt and R. N. Zare, J. Chem. Phys. 48, 4304 (1968).

[22] R. Betti and O. A. Hurricane, Nat. Phys. 12, 435 (2016).

[23] G. G. Balint-Kurti, A. J. Orr-Ewing, J. A. Beswick, A. Brown, and O. S. Vasyutinskii, J. Chem. Phys. 116, 10760 (2002).

[24] A. Brown, G. G. Balint-Kurti, and O. S. Vasyutinskii, J. Phys. Chem. A 108, 7790 (2004).
[25] A. G. Smolin, O. S. Vasyutinskii, G. G. Balint-Kurti, and A. Brown, J. Phys. Chem. A 110, 5371 (2006).

[26] A. Brown, J. Chem. Phys. 122, 084301 (2005).

[27] B. M. Broderick, Y. Lee, M. B. Doyle, V. Y. Chernyak, O. S. Vasyutinskii, and A. G. Suits, Rev. Sci. Instrum. 85, 053103 (2014).

[28] L. Bougas, D. Sofikitis, M. A. Everest, A. J. Alexander, and T. P. Rakitzis, J. Phys. Chem. 133, 174308 (2010).

[29] C. R. Gebhardt, T. P. Rakitzis, P. C. Samartzis, V. Ladopoulos, and T. N. Kitsopoulos, Rev. Sci. Instrum. 72, 3848 (2001).

[30] V. Papadakis and T. N. Kitsopoulos, Rev. Sci. Instrum. 77, 083101 (2006).

[31] A. T. J. B. Eppink and D. H. Parker, Rev. Sci. Instrum. 68, 3477 (1997).

[32] L. D. A. Siebbeles, M. Glass-Maujean, O. S. Vasyutinskii, J. A. Beswick, and O. Roncero, J. Chem. Phys. 100, 3610 (1994).

[33] M. J. Vrakking and S. Stolte, Chem. Phys. Lett. 271, 209 (1997).

[34] R. N. Zare and D. R. Herschbach, Proc. IEEE 51, 173 (1963).

[35] T. P. Rakitzis and R. N. Zare, J. Chem. Phys. 110, 3341 (1999).

[36] T. P. Rakitzis, P. C. Samartzis, R. Toomes, L. Tsigaridas, T. N. Kitsopoulos, M. Coriou, D. Chestakov, A. T. J. B. Eppink, and D. H. Parker, Chem. Phys. Lett. 364, 115 (2002).

[37] D. N. Jodoin and A. Brown, J. Chem. Phys. 123, 054301 (2005).

[38] R. Altkorn, R. N. Zare, and C. H. Greene, Mol. Phys. 55, 1 (1985).

[39] D. Sofikitis, L. Rubio-Lago, M. R. Martin, D. J. A. Brown, N. C.-M. Bartlett, R. N. Zare, and T. P. Rakitzis, Phys. Rev. A 76, 012503 (2007).

[40] N. C.-M. Bartlett, D. J. Miller, R. N. Zare, A. J. Alexander, D. Sofikitis, and T. P. Rakitzis, Phys. Chem. Chem. Phys. 11, 142 (2009).

[41] L. Rubio-Lago, D. Sofikitis, A. Koubenakis, and T. P. Rakitzis, Phys. Rev. A 74, 042503 (2006).

[42] T. P. Rakitzis, Phys. Rev. Lett. 94, 083005 (2005).

[43] J. E. Nafe, E. B. Nelson, and I. I. Rabi, Phys. Rev. 71, 914 (1947).

[44] L. Anderson and T. Walker, Nucl. Instrum. Methods Phys. Res., Sect. A 357, 220 (1995).

[45] A. A. Milner, A. Korobenko, and V. Milner, arXiv:1612 .05328

[46] A. A. Andreev, Y. Guskov, V. I. A. Levkovskii, B. Rozanov, and E. Sherman, J. Exp. Theor. Phys. 92, 69 (2001).

[47] J. Christiansen, D. Ashby, and K. Roberts, Comput. Phys. Commun. 7, 271 (1974).

[48] J. Ayers et al., Proc. SPIE Int. Soc. Opt. Eng. 8505, 85050J (2012).

[49] D. Batani et al., Phys. Scr. T161, 014016 (2014).

[50] H. Paetz gen. Schieck, Eur. Phys. J. A 44, 321 (2010).

[51] K. Lee, J.-O. Hansen, J. F. J. van den Brand, and R. G. Milner, Nucl. Instrum. Methods Phys. Res. 333, 294 (1993).

[52] T. G. Walker and W. Happer, Rev. Mod. Phys. 69, 629 (1997).

[53] S. Karpuk et al., Phys. Part. Nucl. 44, 904 (2013).

[54] J. E. Simmons and R. B. Perkins, Rev. Sci. Instrum. 32, 1173 (1961). 